\title{
GLOBAL EXISTENCE AND UNIQUENESS OF SOLUTIONS FOR A VISCOELASTIC TWO-PHASE MODEL WITH NONLOCAL CAPILLARITY
}

\author{
ALEXANDER DRESSEL \& CHRISTIAN ROHDE
}

\begin{abstract}
The aim of this paper is to study the existence and uniqueness of solutions of an initial-boundary value problem for a viscoelastic two-phase material with capillarity in one space dimension. Therein, the capillarity is modelled via a nonlocal interaction potential. The proof relies on uniform energy estimates for a family of difference approximations: with these estimates at hand we show the existence of a global weak solution. Then, by means of a nontrivial variant of the arguments in [2], uniqueness and optimal regularity are proven. The results of this paper also apply to interaction potentials with non-vanishing negative part and constitute a base for an analysis of the time-asymptotic behaviour.
\end{abstract}

\section{INTRODUCTION}

This paper is concerned with the global existence and uniqueness of weak solutions to the initial value problem

$$
\begin{aligned}
w_{t}-v_{x} & =0 \\
v_{t}-\left[\sigma(w)+L_{\epsilon} w\right]_{x} & =\mu v_{x x}, \\
v(x, 0)=v_{0}(x), \quad w(x, 0) & =w_{0}(x),
\end{aligned}
$$

where the real-valued unknown functions $v=v(x, t)$ and $w=w(x, t)$ of $(x, t) \in$ $[0,1] \times[0, \infty)$ represent the velocity and the strain field in Lagrangian coordinates; we impose either the inhomogeneous Dirichlet boundary condition

$$
\int_{0}^{1} w(y, t) d y=\int_{0}^{1} w_{0}(x) d x \quad \forall t \in[0, \infty)
$$

or the homogeneous Neumann boundary condition

$$
\sigma(w(1, t))+\left(L_{\epsilon} w\right)(1, t)=0 \quad \forall t \in[0, \infty) .
$$

In system (1.1), the diffusion coefficient $\mu>0$ is fixed, and the deformation stress $w \mapsto \sigma(w)$ is given by

$$
\sigma(w)=w^{3}-w
$$

For $\lambda>0$, the capillarity stress $L_{\epsilon}$ is defined by 


$$
L_{\epsilon} u=\lambda\left(\phi_{\epsilon} * u-u\right)
$$

Therein, $u \mapsto \phi_{\epsilon} * u$ is the convolution operator given by

$$
\left(\phi_{\epsilon} * u\right)(x)=\int_{0}^{1} \phi_{\epsilon}(x-y) u(y) d y, \quad \phi_{\epsilon}(x)=\frac{1}{\epsilon} \phi\left(\frac{x}{\epsilon}\right) .
$$

More specific assumptions on the non-local interaction kernel $\phi: \mathbb{R} \rightarrow \mathbb{R}$ will be given in the next section. Here we only note that the interaction kernel is also allowed to have nonvanishing negative part.

The function $\sigma: \mathbb{R} \rightarrow \mathbb{R}$ in (1.5) represents a toy example of a material law for a homogeneous viscoelastic two-phase medium. The stored energy function associated to $\sigma$ is

$$
S(w)=\int_{0}^{w} \sigma(u) d u=\frac{1}{4} w^{4}-\frac{1}{2} w^{2} .
$$

It has the form of a double-well potential with minima at \pm 1 . In case of $w \in$ $(-\infty,-\sqrt{1 / 3})(w \in(\sqrt{1 / 3}, \infty))$ a state $w$ is referred to be in the low (high) strain phase. On the interval $J=(-\sqrt{1 / 3}, \sqrt{1 / 3})$, the transitional region, the function $S^{\prime}=\sigma$ is decreasing.

Due to the double-well structure of $S$, the energy minimizing configuration allows for mixtures of the high and low strain phase, but, at least for nonnegative $\phi_{\epsilon}$ the capillarity stress $L_{\epsilon}$ penalizes sharp interphases. This can be most easily seen from the fact that the evolution process governed by (1.1) dissipates the nonlocal energy

$$
\begin{aligned}
\mathfrak{H}(v, w) & =\int_{0}^{1} S(w(x))+\frac{v(x)^{2}}{2} d x+\mathfrak{E}(w), \\
\mathfrak{E}(w) & =\frac{\lambda}{4} \int_{0}^{1} \int_{0}^{1} \phi_{\epsilon}(x-y)(w(x)-w(y))^{2} d y d x .
\end{aligned}
$$

A local version of (1.9) is given by

$$
\mathfrak{H}_{l o c}(v, w)=\int_{0}^{1} S(w(x))+\frac{v^{2}}{2}+\frac{\gamma \lambda \epsilon^{2}}{2} w_{x}^{2}(x) d x .
$$

This energy is dissipated by the more frequently addressed third-order evolutionary system

$$
\begin{aligned}
w_{t}-v_{x} & =0 \\
v_{t}-\left[\sigma(w)+\gamma \lambda \epsilon^{2} w_{x x}\right]_{x} & =\mu v_{x x}
\end{aligned}
$$

We will show in the next section in more detail how the nonlocal and the local model are connected. In particular we relate the positive constant $\gamma$ in (1.10) and (1.11) to the interaction kernel. Concernig the different modelling background we refer 
to $[6,12,10]$. Here we mention that in contrast to (1.11), solutions of (1.1) do not require more regularity as needed for the solution of (1.1) in the case $\lambda=0$.

Moreover and more important, an advantage of the nonlocal model 1.1 is the fact that ,in case of $\phi_{\epsilon}$ having negative parts, solutions with a finite scale microstructure can develop (cf. [11]) and it is supposed that, in general, the time-asymptotic limit of solutions to (1.1) contains multiple phase transitions induced by the negative part of the kernel. This behaviour cannot be modelled within the approach (1.11).

For non-negative $\phi_{\epsilon}$, the existence of weak solutions to system (1.1) for given initial values (on the spatial domain $\mathbb{R}$ ) was proven for arbitrary Lipschitz continuous $\sigma$ with a variational technique (see [13], Theorem 5.4) relying on the ideas in [4]. This result is extended in this contribution in various directions: initial boundary value problems are addressed, partly negative kernels are treated, and optimal regularity is proven under quite rough initial conditions. Note that we merely assume that the energy of the initial data is bounded.

For the existence of solutions for the initial boundary value problem for (1.11) we refer to [2] and [7] in the case $\lambda=0$. Existence and asymptotic behaviour of solutions to (1.11) with $\lambda \geq 0$ has been studied in [3,8]. Furthermore, for the non-stationary local model, global existence and time-asymptotic convergence to a stationary solution for bounded spatial domains in higher dimension were studied (see [8] and references therein). Note that, in the analysis of the local model, the initial strain $w_{0}$ has to be uniformly bounded.

Let us describe the paper's content in more detail. To obtain a global existence result for weak solutions of our viscoelastic system we will in the first step consider a spatial difference approximation to system (1.1) and obtain uniform energy estimates. With these estimates at hand, we succeed to construct a weak solution to system (1.1).

Then, by a nontrivial variant of the techniques in [2], we prove optimal regularity. Furthermore, for any of these weak solutions, we show by means of a detailed analysis of weak solutions close to $t=0$, also referred to as time layer estimates, that there exists an $L^{\infty}$ bound on $(0,1) \times(\delta, \infty)$ for any $\delta>0$. Note that, in contrast to [2] and [3], the initial strain $w_{0}$ has not to be contained in $L^{\infty}(0,1)$.

Based on the regularity results we obtain uniqueness by a Gronwall argument. In order to apply these techniques, we perform careful Green's function estimates.

The regularity results of this paper will also be used as a base for the analysis of the time-asymptotic behaviour (cf. [5]).

The paper is organized as follows. In Section 2, we specify the assumptions on our non-local model in more detail and relate this model to the local one. In Section 3, we introduce some notation and formulate the main result. In Section 4, we sketch out and perform the proof of the main result.

\section{Specification of the NONLOCAL MODEL AND its RELATiOn TO THE LOCAL ONE}

It is not hard to show that, for $\mu=0$, system (1.1) can be derived from the stationary action principle with the Hamiltonian $\mathfrak{H}$ in (1.9). 
We assume that

$$
\phi \in L^{\infty}(\mathbb{R})
$$

and

$$
\phi(x)=\phi(-x) \forall x \in \mathbb{R} .
$$

From (2.1) and definition (1.6) it follows for any $u \in L^{\infty}(0,1)$

$$
\left\|L_{\epsilon} u\right\|_{\infty} \leq \frac{C}{\epsilon}\|u\|_{\infty}
$$

where $C$ depends on the normalized interaction kernel $\phi$.

Define the bilinear form $B_{\epsilon}$ via

$$
B_{\epsilon}\left(u_{1}, u_{2}\right)=\frac{\lambda}{2} \int_{0}^{1} \int_{0}^{1} \phi_{\epsilon}(x-y)\left(u_{1}(x)-u_{1}(y)\right)\left(u_{2}(x)-u_{2}(y)\right) d y d x
$$

where $\lambda>0$.

For any $\epsilon>0$, there exists a finite constant $C(\epsilon)$ such that

$$
\left|B_{\epsilon}\left(u_{1}, u_{2}\right)\right| \leq C(\epsilon)\left\|u_{1}\right\|_{L^{2}(0,1)}\left\|u_{2}\right\|_{L^{2}(0,1)} \forall u_{1}, u_{2} \in L^{2}(0,1) .
$$

From (1.9) and (2.3) we see that, for any $w \in L^{2}(0,1)$, we have

$$
\mathfrak{E}(w)=\frac{1}{2} B_{\epsilon}(w, w) .
$$

Exploiting the symmetry (2.2), it is not hard to show that, for any $\epsilon>0$, the operator $L_{\epsilon}$ defined in (1.6) fulfills the following relation (cf. [12], p. 112):

$$
\int_{0}^{1} u_{1}(x)\left(L_{\epsilon} u_{2}\right)(x) d x=B_{\epsilon}\left(u_{1}, u_{2}\right) \quad \forall u_{1}, u_{2} \in L^{2}(0,1) .
$$

In this sense, by virtue of relation (2.5), the operator $L_{\epsilon}$ is associated to the nonlocal interaction potential $\mathfrak{E}$.

Finally, we add some comments on the relationship between the local Cahn-Hilliard type (also called Van-der-Waals type) energy and the nonlocal model under the moment assumption

$$
0<\int_{0}^{\infty} x^{n} \phi(x) d x<\infty \text { for } n=2,3 .
$$

In case of $w$ being in $C^{2}([0,1])$, the nonlocal model (1.1) can be related to the local one by means of the following Taylor expansion argument: for $\epsilon$ small there holds 


$$
\begin{aligned}
& \int_{0}^{1} \int_{0}^{1} \phi_{\epsilon}(x-y)(w(x)-w(y))^{2} d y d x \\
& =\int_{0}^{1} \int_{0}^{1} \phi_{\epsilon}(x-y)\left[w_{x}(x)(x-y)+O\left((x-y)^{2}\right)\right]^{2} d y d x
\end{aligned}
$$

and therefore

$$
\int_{0}^{1} \int_{0}^{1} \phi_{\epsilon}(x-y)(w(x)-w(y))^{2} d y d x=I_{1}+I_{2}
$$

where

$$
\begin{aligned}
& I_{1}=\int_{0}^{1} \int_{0}^{1} \phi_{\epsilon}(x-y) w_{x}(x)^{2}(y-x)^{2} d y d x, \\
& I_{2}=\int_{0}^{1} \int_{0}^{1} \phi_{\epsilon}(x-y) O\left(|x-y|^{3}\right) d y d x .
\end{aligned}
$$

Set

$$
\bar{y}:=x-y, \quad \tilde{y}:=\frac{\bar{y}}{\epsilon}
$$

and

$$
M_{\epsilon, x}:=\left\{\frac{x-y}{\epsilon} \in \mathbb{R} \quad \mid \quad y \in[0,1]\right\} .
$$

From definitions (2.9), (2.10) and (2.11) it directly follows

$$
\begin{aligned}
I_{1} & =\int_{0}^{1}\left(\int_{0}^{1} \phi_{\epsilon}(x-y)(x-y)^{2} d y\right) w_{x}(x)^{2} d x \\
& =\int_{0}^{1}\left(\int_{M_{1, x}} \phi_{\epsilon}(\bar{y}) \bar{y}^{2} d \bar{y}\right) w_{x}(x)^{2} d x=\epsilon^{2} \int_{0}^{1}\left(\int_{M_{\epsilon, x}} \phi(\tilde{y}) \tilde{y}^{2} d \tilde{y}\right) w_{x}(x)^{2} d x \\
I_{2} & \left.=\int_{0}^{1} \int_{0}^{1} \phi_{\epsilon}(x-y) O(|x-y|)^{3}\right) d y d x \\
& =\int_{0}^{1} \int_{M_{1, x}} \phi_{\epsilon}(\bar{y}) O\left(|\bar{y}|^{3}\right) d \bar{y} d x=\epsilon^{3} \int_{0}^{1} \int_{M_{\epsilon, x}} \phi(\tilde{y}) O\left(|\tilde{y}|^{3}\right) d \tilde{y} d x .
\end{aligned}
$$

Due to $(2.7)$, for any $x \in[0,1]$, 


$$
\lim _{\epsilon \rightarrow 0} \int_{M_{\epsilon, x}} \phi(\tilde{y}) \tilde{y}^{2} d \tilde{y}=\int_{-\infty}^{0} \phi(\tilde{y}) \tilde{y}^{2} d \tilde{y} \stackrel{(2.1)}{=} \frac{1}{2} \int_{-\infty}^{\infty} \phi(\tilde{y}) \tilde{y}^{2} d \tilde{y}=: \gamma
$$

and

$$
\sup _{\epsilon>0, x \in[0,1]}\left|\int_{0}^{1} \int_{M_{\epsilon, x}} \phi(\tilde{y}) O\left(|\tilde{y}|^{3}\right) d \tilde{y} d x\right|<\infty
$$

In particular,

$$
I_{1}=\epsilon^{2} \gamma \int_{0}^{1} w_{x}(x)^{2} d x+O\left(\epsilon^{3}\right)
$$

Hence, due to $I_{2}=O\left(\epsilon^{3}\right)$ and (2.8), we obtain

$$
\int_{0}^{1} \int_{0}^{1} \phi_{\epsilon}(x-y)(w(x)-w(y))^{2} d y d x=\epsilon^{2} \gamma \int_{0}^{1} w_{x}(x)^{2} d x+O\left(\epsilon^{3}\right) .
$$

We formally obtain the local analogue of the energy (1.9), namely the Cahn-Hilliard (Van-der-Waals) type energy for phase separation given by (1.10) corresponding to system (1.11).

\section{Notation AND MAin THEOREM}

In this section, we formulate the main result about the existence and asymptotic behaviour of weak solutions to system (1.1) with boundary condition (1.3) (resp. (1.4)).

A locally integrable function $(v, w):[0,1] \times[0, \infty) \rightarrow \mathbb{R}^{2}$ is referred to as a weak solution of system (1.1) iff $v, \sigma(w)$ and $L_{\epsilon} w$ are locally integrable on $(0,1) \times(0, \infty)$ and, for any $\psi \in C_{0}^{\infty}((0,1) \times(0, \infty))$,

$$
\begin{aligned}
& \int_{[0, \infty)} \int_{[0,1]}-\psi_{t} w+\psi_{x} v d x d t=0 \\
& \int_{[0, \infty)} \int_{[0,1]}-\psi_{t} v+\psi_{x}\left[\sigma(w)+L_{\epsilon} w\right] d x d t=\int_{[0, \infty)} \int_{[0,1]} \mu \psi_{x x} v d x d t .
\end{aligned}
$$

Before we formulate the main result, we make some remarks on the stored energy $S$ in (1.8), the functional $\mathfrak{E}$ defined in (1.9) and the operator $L_{\epsilon}$ defined in (1.6).

From (1.8) it easily follows

$$
\lim _{|z| \rightarrow \infty} \frac{S(z)}{z^{2}}=\infty
$$

In particular, there exists a finite real number $C$ such that

$$
S(z) \geq C+z^{2} \forall z \in \mathbb{R} .
$$


Furthermore, due to definition (1.9) and relation (2.4), there exists a finite real number $\bar{C}(\epsilon)$ such that

$$
|\mathfrak{E}(w)| \leq \bar{C}(\epsilon)\|w\|_{L^{2}(0,1)}^{2} \forall w \in L^{2}(0,1)
$$

Due to (1.6),

$$
L_{\epsilon} w=-\lambda w+\hat{L}_{\epsilon} w, \text { where } \hat{L}_{\epsilon} w=\lambda \phi_{\epsilon} * w .
$$

The main result of this paper can be formulated as follows:

Theorem 3.1. Assume that, in system (1.1), the scaling parameter $\epsilon$ and the diffusion coefficient $\mu$ are strictly positive, that the initial values $\left(v_{0}, w_{0}\right)$ are Lebesgue-measurable on $(0,1)$ and the functional $\mathfrak{H}$ defined in (1.9) is bounded from above:

$$
\mathfrak{H}\left(v_{0}, w_{0}\right)<\infty
$$

In case of the homogeneous Neumann boundary condition (1.4) additionally assume that

$$
w_{0} \text { is continuous at } x=1 \text { with } \lim _{x \rightarrow 1} \sigma\left(w_{0}(x)\right)+\left(L_{\epsilon} w_{0}\right)(x)=0 .
$$

Then, the following claims are true:

(i). There exists a global weak solution

$$
(v, w) \in C^{0}\left([0, \infty), L^{2}(0,1) \times L^{2}(0,1)\right)
$$

of system (1.1) fulfilling the initial value condition (1.2) and the Dirichlet boundary condition (1.3) (resp. the homogeneous Neumann boundary condition (1.4)). Furthermore, in case of the homogeneous Neumann boundary condition (1.4), for any $t \in[0, \infty), w(\cdot, t)$ is continuous at $x=1$ and

$$
\lim _{x \rightarrow 1} \sigma(w(x, t))+\left(L_{\epsilon} w\right)(x, t)=0 .
$$

(ii). For this weak solution there holds

$$
\begin{gathered}
\mathfrak{H}(v(\cdot, t), w(\cdot, t)) \leq \mathfrak{H}\left(v_{0}, w_{0}\right) \forall t \geq 0, \\
(v, w) \in L^{\infty}\left(\delta, \infty ; H^{1, \infty}(0,1)\right) \times L^{\infty}((0,1) \times(\delta, \infty)) \forall \delta>0
\end{gathered}
$$

and, in case of the Dirichlet boundary condition (1.3),

$$
v \in L^{2}\left(0, \infty ; H_{0}^{1,2}(0,1)\right) .
$$

(iii). On the other hand, any weak solution $(v, w) \in C^{0}\left([0, \infty), L^{2}(0,1) \times L^{2}(0,1)\right)$ to one the aforementioned initial-boundary value problems with (3.5) is unique. 
Remark 3.1. (i). On the initial values $\left(v_{0}, w_{0}\right)$, we only assume that the Hamiltonian $\mathfrak{H}\left(v_{0}, w_{0}\right)$ is bounded. In particular, the initial strain $w_{0}$ has not to be contained in $L^{\infty}(0,1)$ (cf. [2] and [3]).

(ii). In general, the regularity is optimal: it is well known that, for stationary solutions to system (1.1), the strain $w$ may be discontinuous (cf. [1]). As the heat kernel is explicitly given, we obtain optimal regularity for the velocity field $v$ by means of Green's function estimates.

(iii). In contrast to [2], we do not assume $v_{0} \in H_{0}^{1,2}(0,1)$. In fact, we only assume $v_{0} \in$ $L^{2}(0,1)$ and we will see that, under the Dirichlet boundary condition (1.3), the weak solution $(v, w)$ constructed below a posteriori is contained in $C^{0}\left([0, \infty), L^{2}(0,1) \times L^{2}(0,1)\right)$ and fulfills relation (3.7). These conditions together with (1.3) imply uniqueness for the Dirichlet case. The treatment of the homogeneous Neumann boundary condition will be reduced to the Dirichlet case condition by means of a mirroring argument.

\section{Proof of MAIN TheOREM}

The proof is subdivided into six parts:

(i) uniform energy estimates on a family of difference approximations for the Dirichlet boundary condition (1.3),

(ii) construction of a weak solution as a weak accumulation point of this family in an appropriate space,

(iii) analysis of this weak solution close to $t=0$ (time layer estimates),

(iv) proof of uniqueness,

(v) Green's function estimates on the velocity field $v$,

(vi) treatment of the homogeneous Neumann boundary condition (1.4).

Remark 4.1. In fact, we first prove the existence, regularity and uniqueness of a weak solution to the Dirichlet boundary condition (1.3) and then, in part (vi), apply a mirroring argument in order to construct a solution to the homogeneous Neumann boundary condition (1.4).

4.1. Energy estimates on difference approximations. In order to construct the difference approximations in part (i) of the proof, we introduce some notation.

Notation 4.1. For an integer $n \geq 2$, define $h=\frac{1}{n-1}$, and, for $i=1, \cdots, n$, set $x_{i}=(i-1) h$. For an $(n-1)$-vector $\mathbf{u}$, let $P_{0}^{(n)} \mathbf{u}:[0,1] \rightarrow \mathbb{R}$ denote the piecewise constant function with $\left(P_{0}^{(n)} \mathbf{u}\right)(y)=\mathbf{u}_{1}$ for $0=x_{1} \leq y \leq x_{2}$ and $\left(P_{0}^{(n)} \mathbf{u}\right)(y)=\mathbf{u}_{i}$ for $x_{i}<y \leq x_{i+1},(i=2, \cdots, n-1)$. Furthermore, for an $n$-vector $\mathbf{u}$, let $P_{1}^{(n)} \mathbf{u}:[0,1] \rightarrow \mathbb{R}$ denote the piecewise linear function with $\left(P_{1}^{(n)} \mathbf{u}\right)\left(x_{i}\right)=\mathbf{u}_{i}$ for $i=1, \cdots, n$.

In this subsection, for fixed $n \in \mathbb{N}$, set

$$
\mathbb{X}_{n}:=\left\{\mathbf{u} \in \mathbb{R}^{n} \quad \mid \mathbf{u}_{1}=\mathbf{u}_{n}=0\right\}
$$

and, for $i=1,2$, write $P_{i}$ instead of $P_{i}^{(n)}$.

Consider the following initial value problem for $(\mathbf{v}, \mathbf{w}):[0, \infty) \rightarrow \mathbb{R}^{n} \times \mathbb{R}^{n-1}$ : 


$$
\begin{gathered}
\mathbf{w}_{t}-\delta_{h} \mathbf{v}=0, \\
\mathbf{v}_{t}-\bar{\delta}_{h} \mathbf{g}(\mathbf{w})=\mu \bar{\delta}_{h} \delta_{h} \mathbf{v}, \\
(\mathbf{v}(0), \mathbf{w}(0))=\left(\mathbf{v}_{0}, \mathbf{w}_{0}\right) \in \mathbb{X}_{n} \times \mathbb{R}^{n-1} .
\end{gathered}
$$

Therein, for any $\mathbf{u} \in \mathbb{R}^{n-1}$, the mapping $\mathbf{u} \mapsto \mathbf{g}(\mathbf{u})$ is defined by

$$
\mathbf{g}(\mathbf{u})_{i}=\sigma\left(\mathbf{u}_{i}\right)+\left(\mathbf{L}_{\epsilon}(\mathbf{u})\right)_{i}
$$

where $\sigma$ is given in (1.5) and $\mathbf{L}_{\epsilon}: \mathbb{R}^{n-1} \rightarrow \mathbb{R}^{n-1}$ is set to

$$
\left(\mathbf{L}_{\epsilon}(\mathbf{u})\right)_{i}=\left(L_{\epsilon}\left(P_{0} \mathbf{u}\right)\right)\left(x_{i}\right) .
$$

For any $\mathbf{u} \in \mathbb{X}_{n}, \delta_{h} \mathbf{u} \in \mathbb{R}^{n-1}$ denotes the forward difference quotient defined by

$$
\left(\delta_{h} \mathbf{u}\right)_{i}=\frac{\mathbf{u}_{i+1}-\mathbf{u}_{i}}{h} \text { for } i=1, \cdots, n-1,
$$

and, for any $\mathbf{u} \in \mathbb{R}^{n-1}$, set

$$
\begin{aligned}
\left(\bar{\delta}_{h} \mathbf{u}\right)_{1} & =\left(\bar{\delta}_{h} \mathbf{u}\right)_{n}=0 \\
\left(\bar{\delta}_{h} \mathbf{u}\right)_{i} & =\frac{\mathbf{u}_{i}-\mathbf{u}_{i-1}}{h} \text { for } i=2, \cdots, n-1 .
\end{aligned}
$$

System (4.2) is our spatial discretization of system (1.1) to step-size $h$.

Due to the classical existence theory for ODE, for some $T>0$, there exists a solution of the initial value problem (4.2) on the time interval $[0, T]$ with

$$
\left.(\mathbf{v}(\cdot), \mathbf{w}(\cdot)) \in C^{1}\left([0, T], \mathbb{R}^{n} \times \mathbb{R}^{n-1}\right), \quad(\mathbf{v}(0), \mathbf{w}(0))=\left(\mathbf{v}_{0}, \mathbf{w}_{0}\right)\right) .
$$

Due to definition (4.6),

$$
\operatorname{Range}\left(\bar{\delta}_{h}\right) \subset \mathbb{X}_{n}
$$

According to (4.8), (4.2) and (4.3),

$$
\mathbf{v}(t)=\mathbf{v}_{0}+\int_{0}^{t} \bar{\delta}_{h}\left(\mathbf{g}(\mathbf{w}(s))+\mu \delta_{h} \mathbf{v}(s)\right) d s \in \mathbb{X}_{n} \forall t \in[0, T]
$$

In particular,

$$
\mathbf{v}_{1}(t)=\mathbf{v}_{n}(t)=0 \quad \forall t \in[0, T]
$$

By definition (4.5), for any $\mathbf{u} \in \mathbb{X}_{n}$,

$$
P_{0}\left(\delta_{h} \mathbf{u}\right)=\partial_{x}\left(P_{1} \mathbf{u}\right)
$$


in the weak sense and therefore

$$
h\left|\delta_{h} \mathbf{u}\right|^{2}=\int_{0}^{1} \partial_{x}\left(P_{1} \mathbf{u}\right)(x)^{2} d x \quad \forall \mathbf{u} \in \mathbb{X}_{n}
$$

Recall the definition of $P_{0}=P_{0}^{(n)}$ in Notation 4.1 and obtain for any $C^{1}$ solution $(\mathbf{v}, \mathbf{w})=(\mathbf{v}(t), \mathbf{w}(t))$ of $(4.2)$ :

$$
\begin{gathered}
\frac{d}{d t} \int_{0}^{1}\left(P_{0} \mathbf{w}(t)\right)(x) d x=h \frac{d}{d t} \sum_{i=1}^{n-1}(\mathbf{w}(t))_{i}=h \sum_{i=1}^{n-1}\left(\mathbf{w}_{t}(t)\right)_{i} \\
\stackrel{(4.2)}{=} h \sum_{i=1}^{n-1}\left(\delta_{h} \mathbf{v}(t)\right)_{i} \stackrel{(4.5)}{=} \sum_{i=1}^{n-1} \mathbf{v}_{i+1}(t)-\mathbf{v}_{i}(t)=\mathbf{v}_{n}(t)-\mathbf{v}_{1}(t) \stackrel{(4.9)}{=} 0 .
\end{gathered}
$$

In particular,

$$
\int_{0}^{1}\left(P_{0} \mathbf{w}(t)\right)(x) d x=\int_{0}^{1}\left(P_{0} \mathbf{w}(0)\right)(x) d x \quad \forall t \in[0, \infty) .
$$

In this sense, the difference approximation is consistent with the displacement boundary condition (1.3).

Due to definitions (4.1) and (4.8),

$$
\mathbf{x}_{1}=\mathbf{x}_{n}=\left(\bar{\delta}_{h} \mathbf{y}\right)_{1}=\left(\bar{\delta}_{h} \mathbf{y}\right)_{n}=0 \quad \forall(\mathbf{x}, \mathbf{y}) \in \mathbb{X}_{n} \times \mathbb{R}^{n-1} .
$$

In particular, for any $(\mathbf{x}, \mathbf{y}) \in \mathbb{X}_{n} \times \mathbb{R}^{n-1}$,

$$
\begin{aligned}
\mathbf{x} \cdot\left(\bar{\delta}_{h} \mathbf{y}\right) & =\sum_{i=1}^{n} \mathbf{x}_{i}\left(\bar{\delta}_{h} \mathbf{y}\right)_{i} \stackrel{(4.13)}{=} \sum_{i=2}^{n-1} \mathbf{x}_{i}\left(\bar{\delta}_{h} \mathbf{y}\right)_{i} \stackrel{(4.6)}{=} \sum_{i=2}^{n-1} \mathbf{x}_{i} \frac{\mathbf{y}_{i}-\mathbf{y}_{i-1}}{h} \\
& =\sum_{i=2}^{n-1} \frac{\mathbf{x}_{i} \mathbf{y}_{i}}{h}-\frac{\mathbf{x}_{i} \mathbf{y}_{i-1}}{h}=\sum_{i=2}^{n-1} \frac{\mathbf{x}_{i} \mathbf{y}_{i}}{h}-\sum_{i=1}^{n-2} \frac{\mathbf{x}_{i+1} \mathbf{y}_{i}}{h} \\
& \stackrel{(4.13)}{=} \sum_{i=1}^{n-1} \frac{\mathbf{x}_{i} \mathbf{y}_{i}}{h}-\sum_{i=1}^{n-1} \frac{\mathbf{x}_{i+1} \mathbf{y}_{i}}{h}=-\sum_{i=1}^{n-1} \frac{\mathbf{x}_{i+1}-\mathbf{x}_{i}}{h} \mathbf{y}_{i} \\
& \stackrel{(4.5)}{=}-\left(\delta_{h} \mathbf{x}\right) \cdot \mathbf{y},
\end{aligned}
$$

and we obtain

$$
\mathbf{x} \cdot\left(\bar{\delta}_{h} \mathbf{y}\right)=-\left(\delta_{h} \mathbf{x}\right) \cdot \mathbf{y} \quad \forall(\mathbf{x}, \mathbf{y}) \in \mathbb{X}_{n} \times \mathbb{R}^{n-1} .
$$

Recall the definition (1.8) of the stored energy function $S$ and set 


$$
\mathbf{E}_{\epsilon, n}(\mathbf{v}, \mathbf{w}):=h\left[\sum_{i=1}^{n-1} S\left(\mathbf{w}_{i}\right)+\frac{1}{2} \mathbf{v}_{i}^{2}\right]+\mathbf{B}_{\epsilon}(\mathbf{w}, \mathbf{w}) .
$$

In particular,

$$
\frac{d}{d t} \mathbf{E}_{\epsilon, n}(\mathbf{v}, \mathbf{w})=h \mathbf{g}(\mathbf{w}) \cdot \mathbf{w}_{t}+h \mathbf{v} \cdot \mathbf{v}_{t}
$$

Therein, $\mathbf{B}_{\epsilon}$ is the discrete analogue to the bilinear form defined in (2.3):

$$
\mathbf{B}_{\epsilon}\left(\mathbf{u}_{1}, \mathbf{u}_{2}\right)=\frac{1}{2} \mathbf{u}_{1} \cdot \mathbf{L}_{\epsilon} \mathbf{u}_{2} .
$$

The energy $\mathbf{E}_{\epsilon, n}$ defined in (4.15) is the discretized analogue of the Hamiltonian $\mathfrak{H}$ in (1.9).

The aim of this section is to prove the following lemma:

Lemma 4.2. For any $\left(\mathbf{v}_{0}, \mathbf{w}_{0}\right) \in \mathbb{X}_{n} \times \mathbb{R}^{n-1}$, a solution

$$
(\mathbf{v}(\cdot), \mathbf{w}(\cdot)) \in C^{1}\left([0, \infty) ; \mathbb{X}_{n} \times \mathbb{R}^{n-1}\right)
$$

of the initial-value problem (4.2)-(4.3) exists globally in time. More precisely, the mapping

$$
t \mapsto\left(P_{1} \mathbf{v}(t), P_{0} \mathbf{w}(t)\right),
$$

is uniformly bounded in $C^{0}\left([0, \infty) ; L^{2}(0,1) \times L^{2}(0,1)\right)$ by a constant $C=C\left(\mathfrak{H}\left(P_{1} \mathbf{v}_{0}, P_{0} \mathbf{w}_{0}\right)\right)$, and, for any $t>0$,

$$
\frac{d}{d t} \mathbf{E}_{\epsilon, n}(\mathbf{v}(t), \mathbf{w}(t))=-\mu \int_{0}^{1} \partial_{x}\left(P_{1} \mathbf{v}(t)\right)(x)^{2} d x \leq 0 .
$$

Furthermore, there exists a finite real number $K=K\left(\mathfrak{H}\left(P_{1} \mathbf{v}_{0}, P_{0} \mathbf{w}_{0}\right)\right)$ such that

$$
\mathbf{E}_{\epsilon, n}(\mathbf{v}(t), \mathbf{w}(t)) \geq K+\left\|P_{0} \mathbf{w}(t)\right\|_{L^{2}(0,1)} \forall t \in(0, \infty) .
$$

Proof of Lemma 4.2: Our aim is to derive an energy estimate for the solution $(\mathbf{v}(\cdot), \mathbf{w}(\cdot))$ of (4.2) with (4.7). With this estimate at hand, we will prove global existence by means of a bootstrap argument.

Due to (4.14), we have

$$
\mathbf{g}(\mathbf{w}) \cdot \delta_{h} \mathbf{v}+\mathbf{v} \cdot \bar{\delta}_{h} \mathbf{g}(\mathbf{w})=\mathbf{g}(\mathbf{w}) \cdot \delta_{h} \mathbf{v}-\mathbf{g}(\mathbf{w}) \cdot \delta_{h} \mathbf{v}=0 .
$$

Scalar multiplication of $h \mathbf{g}(\mathbf{w})$ (resp. $h \mathbf{v}$ ) to the first (resp. second) equation in (4.2) from the left, addition of the resulting two equations and exploiting relation (4.20) give 


$$
h \mathbf{g}(\mathbf{w}) \cdot \mathbf{w}_{t}+h \mathbf{v} \cdot \mathbf{v}_{t}=h \mu \mathbf{v} \cdot\left(\bar{\delta}_{h} \delta_{h} \mathbf{v}\right)=-h \mu\left|\delta_{h} \mathbf{v}\right|^{2} \leq 0 .
$$

Relation (4.21) together with (4.11) and (4.16) implies relation (4.18).

It remains to verify relation (4.19).

From (4.15) we conclude that

$$
\mathbf{E}_{\epsilon, n}(\mathbf{v}(t), \mathbf{w}(t)) \geq \mathbf{B}_{\epsilon}(\mathbf{w}(t), \mathbf{w}(t))+\int_{0}^{1} \frac{1}{4}\left(P_{0} \mathbf{w}(t)\right)(x)^{4}-\frac{1}{2}\left(P_{0} \mathbf{w}(t)\right)(x)^{2}
$$

where $\mathbf{B}_{\epsilon}$ is the bilinear form in (4.17).

There holds for some constant $C=C(\epsilon)$

$$
\left|\mathbf{B}_{\epsilon}(\mathbf{w}(t), \mathbf{w}(t))\right| \leq C\left\|P_{0} \mathbf{w}(t)\right\|_{L^{2}(0,1)}^{2}
$$

and, for any $\delta>0$, we have

$$
\int_{0}^{1}\left(P_{0} \mathbf{w}\right)(x)^{2} d x \leq \delta+\frac{1}{4 \delta} \int_{0}^{1}\left(P_{0} \mathbf{w}\right)(x)^{4} d x .
$$

Hence, relation (4.19) follows and Lemma 4.2 has been proven.

4.2. Construction of weak solution. Recall the definition of $P_{0}^{(n)}$ and $P_{1}^{(n)}$ in Notation 4.1 and let $\left\{\left(P_{1}^{(n)} \mathbf{v}(\cdot), P_{0}^{(n)} \mathbf{w}(\cdot)\right)\right\}_{n \in \mathbb{N}}$ be a sequence of solutions of (4.2) in $C^{1}\left([0, \infty), L^{2}(0,1) \times L^{2}(0,1)\right)$ with

$$
\lim _{n \rightarrow \infty}\left(P_{1}^{(n)} \mathbf{v}_{0}, P_{0}^{(n)} \mathbf{w}_{0}\right)=\left(v_{0}, w_{0}\right) \text { in } L^{2}(0,1)
$$

such that

$$
\int_{0}^{1}\left(P_{0}^{(n)} \mathbf{w}_{0}\right)(x) d x=\int_{0}^{1} w_{0}(x) d x \quad \forall n \in \mathbb{N} .
$$

Such a sequence exists: with $\left(\mathbf{w}_{0}\right)_{i}$ being set to

$$
\left(\mathbf{w}_{0}\right)_{i}=\frac{1}{x_{i+1}-x_{i}} \int_{x_{i}}^{x_{i+1}} w_{0}(x) d x \text { for } i=1, \cdots n-1,
$$

(where $x_{i}$ is defined in Notation 4.1) relation (4.22) is fulfilled.

According to Lemma 4.2 there exists a finite real number $C\left(\epsilon, v_{0}, w_{0}\right)$ (also not depending on $n$ ) such that

$$
\mathbf{E}_{\epsilon, n}(\mathbf{v}(t), \mathbf{w}(t)) \leq C\left(\epsilon, v_{0}, w_{0}\right) \forall t \in[0, \infty) .
$$

From (4.18) we obtain 


$$
\begin{aligned}
& \mathbf{E}_{\epsilon, n}(\mathbf{v}(0), \mathbf{w}(0))-\mathbf{E}_{\epsilon, n}(\mathbf{v}(\infty), \mathbf{w}(\infty)) \\
& =\mu \int_{0}^{\infty} \int_{0}^{1} \partial_{x}\left(P_{1}^{(n)} \mathbf{v}(t)\right)(x)^{2} d x d t \\
& =\mu\left\|\partial_{x}\left(P_{1}^{(n)} \mathbf{v}(\cdot)\right)\right\|_{L^{2}([0, \infty) \times[0,1])}^{2} .
\end{aligned}
$$

Note that, due to (4.19), the mapping

$$
t \mapsto \mathbf{E}_{\epsilon, n}(\mathbf{v}(t), \mathbf{w}(t))
$$

is bounded from below. Furthermore, due to (4.18), this mapping is monotone in $t$.

From (4.23) and (4.24) we conclude that the sequence $\left\{P_{1}^{(n)} \mathbf{v}(\cdot)\right\}_{n \in \mathbb{N}}$ is bounded in $L^{2}\left(0, \infty ; H_{0}^{1,2}(0,1)\right)$ by a constant $K\left(\mu, v_{0}, w_{0}\right)$. Hence, from the weak lower semicontinuity of the $L^{2}\left(0, \infty ; H^{1,2}(0,1)\right)$ - norm we conclude that, for any weak accumulation point $v \in L^{2}\left(0, \infty ; H_{0}^{1,2}(0,1)\right)$ of the sequence $\left\{P_{1}^{(n)} \mathbf{v}\right\}_{n \in \mathbb{N}}$,

$$
\int_{0}^{\infty} \int_{0}^{1} v(x, t)^{2}+v_{x}(x, t)^{2} d x d t \leq K\left(\mu, v_{0}, w_{0}\right) .
$$

Due to Lemma 4.2, for any finite $T>0$, the sequence $\left\{P_{0}^{(n)} \mathbf{w}\right\}_{n \in \mathbb{N}}$ has a weak accumulation point in $w \in L^{2}\left(0, T ; L^{2}(0,1)\right)$.

Without loss of generality, we can assume that $\left\{\left(P_{1}^{(n)} \mathbf{v}, P_{0}^{(n)} \mathbf{w}\right)\right\}_{n \in \mathbb{N}}$ weakly converges in $L^{2}\left(0, T ; L^{2}(0,1)\right)$.

In particular, the spatial integral of $\left(P_{1}^{(n)} \mathbf{v}, P_{0}^{(n)} \mathbf{w}\right)$ strongly converges for $n \rightarrow \infty$, and, as, due to the construction of the difference approximations,

$$
\int_{0}^{1}\left(P_{0}^{(n)} \mathbf{w}\right)(x, t) d x=\int_{0}^{1} w_{0}(x) d x \quad \forall n \in \mathbb{N},
$$

we conclude that, for any finite $T>0$, the weak limit $(v, w)$ solves $(1.1)$, and, at any Lebesgue point $t \in(0, \infty)$ of $t \mapsto w(\cdot, t)$ in $L^{2}\left(0, T ; L^{2}(0,1)\right)$ there holds

$$
\int_{0}^{1} w(x, t) d x=\int_{0}^{1} w_{0}(x) d x
$$

By means of a similar argument, we conclude that, at any Lebesgue point $t \in(0, \infty)$ of $t \mapsto v(\cdot, t)$ in $L^{2}\left(0, \infty ; H^{1,2}(0,1)\right)$, the traces $v(0, t)$ and $v(1, t)$ of the weak limit point $v$ are defined and

$$
v(0, t)=v(1, t)=0 \text { for almost all } t \in(0, \infty)
$$

In particular, 


$$
v \in L^{2}\left(0, \infty ; H_{0}^{1,2}(0,1)\right) .
$$

Now, we will show relation (3.3) for the weak solution $(v, w)$. In particular, it fulfills the initial-value problem in the sense that the evaluation

$$
(v, w) \mapsto(v(\cdot, 0), w(\cdot, 0))=\left(v_{0}, w_{0}\right)
$$

is well defined.

From (4.25) and the first equation in (1.1) we conclude that

$$
v_{x}, w_{t} \in L^{2}\left(0, \infty ; L^{2}(0,1)\right) .
$$

From $w_{0} \in L^{2}(0,1)$ and $(4.28)$ we conclude, that, for any $t \in(0, \infty)$, the integral

$$
w(\cdot, t)=w_{0}+\int_{0}^{t} w_{t}(\cdot, s) d s
$$

is contained in $L^{2}(0,1)$ and that

$$
w \in C^{0}\left([0, \infty) ; L^{2}(0,1)\right) .
$$

From Green's function estimates in Section 4.6 we easily obtain that

$$
v \in C^{0}\left([0, \infty) ; L^{2}(0,1)\right)
$$

and we have proven part (i) of Theorem 3.1 under the Dirichlet boundary condition (1.3).

Furthermore, after multiplying $\sigma(w(x, t))+\left(L_{\epsilon} w\right)(x, t)$ to the first and $v(x, t)$ to the second equation in (1.1) and spatial integration over $(0,1)$, and exploiting relation (4.26), we obtain for a.e. $t \in(0, \infty)$

$$
\int_{0}^{1}\left(\sigma(w(x, t))+\left(L_{\epsilon} w\right)(x, t)\right) w_{t}(x, t)+v(x, t) v_{t}(x, t)=-\mu \int_{0}^{1} v_{x}(x, t)^{2} d x \leq 0 .
$$

Recall relations (2.5), (2.6) and that, due to (1.8), we have $S^{\prime}=\sigma$.

Hence, we obtain for the Hamiltonian $\mathfrak{H}$ defined in $(1.9)$ for a.e. $t \in(0, \infty)$

$$
\frac{d}{d t} \mathfrak{H}(v(\cdot, t), w(\cdot, t)) \leq 0
$$

and relation (3.5) follows. 
4.3. $L^{\infty}$ estimates on the strain. The aim of this section is to prove relation (3.6) for any $\delta>0$ by means of appropriate time-layer estimates.

Recall that $S=S(w)$ denotes the stored energy function defined in (1.8). Furthermore, for any $\left(z_{1}, z_{2}\right) \in \mathbb{R}^{2}$ let $\bar{\sigma}$ fulfill the following relation

$$
\bar{\sigma}\left(z_{1}, z_{2}\right)\left(z_{1}-z_{2}\right)=\sigma\left(z_{1}\right)-\sigma\left(z_{2}\right)
$$

A straightforward calculation gives

$$
\bar{\sigma}\left(z_{1}, z_{2}\right)=z_{1}^{2}+z_{1} z_{2}+z_{2}^{2}-1
$$

and, for its partial derivatives,

$$
\bar{\sigma}_{z_{1}}\left(z_{1}, z_{2}\right)=2 z_{1}+z_{2}, \quad \bar{\sigma}_{z_{2}}\left(z_{1}, z_{2}\right)=2 z_{2}+z_{1} .
$$

The $L^{\infty}(0,1)$-bound of $w(\cdot, t)$ for $t \geq \delta>0$ of the main theorem under the Dirichlet boundary condition (1.3) follows from the following lemma.

Lemma 4.3. Under the assumptions of Theorem 3.1 and the Dirichlet boundary condition (1.3), for any $\delta>0$, the function $(x, t) \mapsto w(x, t)$ is contained in $L^{\infty}((0,1) \times$ $(\delta, \infty))$.

Proof: For $x, y \in[0,1]$ set

$$
q(x, y, t):=\mu(w(y, t)-w(x, t))+\int_{y}^{x} v(z, t) d z .
$$

From definition (4.33) it follows immediately, that, for

we have

$$
a(x, y, t)=\int_{y}^{x} v(z, t) d z
$$

$$
w(x, t)=\frac{1}{\mu}[a(x, y, t)-q(x, y, t)]+w(y, t) .
$$

In the first step, we will prove the following differential equality:

$$
\begin{aligned}
& \frac{d}{d t} S(q(x, y, t)) \\
& =\sigma(q(x, y, t)) \times\left(\left(\hat{L}_{\epsilon} w\right)(x, t)-\left(\hat{L}_{\epsilon} w\right)(y, t)-\frac{\lambda}{\mu}(a(x, y, t)-q(x, y, t))\right. \\
& \left.\quad+\sigma\left(\frac{1}{\mu}[a(x, y, t)-q(x, y, t)]+w(y, t)\right)-\sigma(w(y, t))\right) .
\end{aligned}
$$


From $v \in L^{\infty}\left(0, \infty ; L^{2}(0,1)\right)$ we know that there exists a constant $K>0$ not depending on $(x, y, t)$ such that

$$
|a(x, y, t)| \leq K
$$

Due to (4.29), there exists a point $y \in(0,1)$ and a strictly positive finite real number $C$ such that

$$
|w(y, t)| \leq C \text { a.e. on }(0,1)
$$

For $x \in[0,1]$ and $t \in[0, \infty)$ set

$$
V(x, t)=\int_{0}^{x} v(z, t) d z=a(x, 0, t)
$$

By integration of the second equation of (1.1) in the spatial variable we get for almost every $t \in(0, \infty)$ and $x, y \in(0,1)$

$$
\begin{aligned}
\frac{d}{d t} \int_{y}^{x} v(z, t) d z= & V_{t}(x, t)-V_{t}(y, t) \\
= & \sigma(w(x, t))+\left(L_{\epsilon} w\right)(x, t)+\mu v_{x}(x, t) \\
& -\left(\sigma(w(y, t))+\left(L_{\epsilon} w\right)(y, t)+\mu v_{x}(y, t)\right) .
\end{aligned}
$$

Recalling also the first equation of (1.1) we summarize for convenience of the reader:

$$
\begin{aligned}
w_{t} & =v_{x} \text { in }(0,1) \\
\frac{d}{d t} \int_{z=y}^{x} v(z, t) d z= & \sigma(w(x, t))+\left(L_{\epsilon} w\right)(x, t)+\mu v_{x}(x, t) \\
& -\left(\sigma(w(y, t))+\left(L_{\epsilon} w\right)(y, t)+\mu v_{x}(y, t)\right) .
\end{aligned}
$$

With these identities at hand, we get for almost all $t \in(0, \infty)$ and $x, y \in(0,1)$ 


$$
\begin{aligned}
\frac{d}{d t} q(x, y, t) \stackrel{(4.33)}{=} \mu\left(w_{t}(y, t)-w_{t}(x, t)\right) & +\frac{d}{d t} \int_{z=y}^{x} v(z, t) d z \\
& \quad-\left(\sigma(w(y, t))+\left(L_{\epsilon} w\right)(y, t)+\mu v_{x}(y, t)\right) \\
\stackrel{(4.36)}{=} \mu\left(v_{x}(y, t)-v_{x}(x, t)\right) & +\sigma(w(x, t))+\left(L_{\epsilon} w\right)(x, t)+\mu v_{x}(x, t) \\
= & \sigma(w(x, t))+\left(L_{\epsilon} w\right)(x, t)-\left(\sigma(w(y, t))+\left(L_{\epsilon} w\right)(y, t)\right) \\
& \stackrel{(4.34)}{=} \frac{\lambda}{\mu}(q(x, y, t)-a(x, y, t))+\left(\hat{L}_{\epsilon} w\right)(x, t)-\left(\hat{L}_{\epsilon} w\right)(y, t) \\
& +\sigma\left(\frac{1}{\mu}(a(x, y, t)-q(x, y, t))+w(y, t)\right)-\sigma(w(y, t))
\end{aligned}
$$

where $\hat{L}_{\epsilon}$ is given by relation $(3.2)$.

In particular, relation (4.35) follows for almost all $t \in(0, \infty)$ and $x, y \in(0,1)$.

In the second step, by means of relation (4.35), we will show that there exist real numbers $\hat{K}$ and $\tilde{K}$ depending on the initial values $\left(v_{0}, w_{0}\right)$ such that

$$
|q(x, y, t)| \geq \hat{K} \Rightarrow \frac{d}{d t}(S(q(x, y, t))) \leq-\tilde{K}|S(q(x, y, t))|^{\frac{3}{2}} .
$$

From $w \in L^{\infty}\left(0, \infty ; L^{2}(0,1)\right)$ we conclude that

$$
\sup _{(x, y, t) \in(0,1) \times(0,1) \times(0, \infty)}\left\|\left(\hat{L}_{\epsilon} w\right)(x, t)-\left(\hat{L}_{\epsilon} w\right)(y, t)\right\|_{L^{2}(0,1)}<\infty .
$$

Recall from (1.5) that

$$
\sigma(z)=z^{3}-z .
$$

Let $c_{1}, c_{2}$ and $c_{3}$ be given real numbers. Then, we obtain

$$
\sigma(z)\left(\sigma\left(c_{1}-z\right)+c_{2}+c_{3} z\right)=-z^{6}+P\left(c_{1}, c_{2}, c_{3}, z\right),
$$

where $z \mapsto P\left(c_{1}, c_{2}, c_{3}, z\right)$ is a fifth-order polynomial function with coefficients depending on $c_{1}, c_{2}$ and $c_{3}$.

From (4.39) it easily follows that there exist strictly positive real numbers $\hat{K}=$ $\hat{K}\left(\left|c_{1}\right|,\left|c_{2}\right|,\left|c_{3}\right|\right)$ and $\tilde{K}=\tilde{K}\left(\left|c_{1}\right|,\left|c_{2}\right|,\left|c_{3}\right|\right)$ (where $\hat{K}$ and $\tilde{K}$ are monotone increasing in $\left|c_{i}\right|$ for any $\left.i \in\{1,2,3\}\right)$ such that 


$$
|z| \geq \hat{K} \Rightarrow \sigma(z)\left(\sigma\left(c_{1}-z\right)+c_{2}+c_{3} z\right) \leq-\tilde{K}|S(z)|^{\frac{3}{2}}
$$

In particular, the negative contribution from the $z^{6}$-term on the right-hand side of relation (4.39) dominates in the following sense:

$$
|z| \geq \hat{K} \Rightarrow \sigma(z)\left(\sigma\left(c_{1}-z\right)+c_{2}+c_{3} z\right)<0 .
$$

Due to $(4.35),(4.38)$ and $(4.40)$, relation (4.37) follows for some strictly positive real numbers $\hat{K}$ and $\tilde{K}$.

The $L^{\infty}$ bound now easily follows from Lemma 4.4 below.

Lemma 4.4. Let $\alpha, C$ and $y_{0}$ be strictly positive real numbers with $\alpha \in(1, \infty)$. Then, any solution of the differential inequality

$$
\frac{d}{d t} y \leq-C|y|^{\alpha}, \quad y(0)=y_{0}
$$

is majorized by the solution of the differential equality

given by

$$
\frac{d}{d t} \bar{y}=-C \bar{y}^{\alpha}, \quad \bar{y}(0)=y_{0}
$$

$$
\bar{y}(t)=C(\alpha-1)\left(t+\left(\frac{C(\alpha-1)}{y_{0}}\right)^{\alpha-1}\right)^{\frac{-1}{\alpha-1}} .
$$

Proof: elementary calculus $\square$

4.4. Uniqueness. In this section, we prove part (iii) of Theorem 3.1 under the Dirichlet boundary condition (1.3).

For $i=1,2$ let $\left(w^{(i)}, v^{(i)}\right) \in C^{0}\left([0, \infty) ; L^{2}(0,1)\right)$ be weak solutions of (1.1) with

$$
\sup _{t \in[0, \infty)} \mathfrak{H}\left(w^{(i)}(\cdot, t), v^{(i)}(\cdot, t)\right)<\infty \text { for } i=1,2
$$

and

$$
w^{(1)}(\cdot, 0)-w^{(2)}(\cdot, 0)=v^{(1)}(\cdot, 0)-v^{(2)}(\cdot, 0)=0 .
$$

Then,

$$
(\Delta, \tilde{\Delta}):=\left(w^{(1)}-w^{(2)}, v^{(1)}-v^{(2)}\right)
$$

is a weak solution of the following system:

$$
\begin{aligned}
\Delta_{t}-\tilde{\Delta}_{x} & =0 \\
\tilde{\Delta}_{t}-\left[\bar{\sigma}\left(w^{(1)}, w^{(2)}\right) \Delta+L_{\epsilon} \Delta\right]_{x} & =\mu \tilde{\Delta}_{x x}
\end{aligned}
$$


where $\bar{\sigma}$ is given by $(4.31)$.

We have for any $\theta>0$

$$
\begin{gathered}
\int_{0}^{1}\left[\left(\theta+\bar{\sigma}\left(w^{(1)}, w^{(2)}\right)\right) \Delta+L_{\epsilon} \Delta\right] \tilde{\Delta}_{x}+\left[\bar{\sigma}\left(w^{(1)}, w^{(2)}\right) \Delta+L_{\epsilon} \Delta\right]_{x} \tilde{\Delta} d x \\
\stackrel{(3.7)}{=} \int_{0}^{1}\left[\left(\bar{\sigma}\left(w^{(1)}, w^{(2)}\right)\right) \Delta+L_{\epsilon} \Delta\right] \tilde{\Delta}_{x}-\left[\bar{\sigma}\left(w^{(1)}, w^{(2)}\right) \Delta+L_{\epsilon} \Delta\right] \tilde{\Delta}_{x}+\theta \Delta \tilde{\Delta}_{x} d x=\int_{0}^{1} \theta \Delta \tilde{\Delta}_{x} d x .
\end{gathered}
$$

Hence, after multiplying $\left(\theta+\bar{\sigma}\left(w^{(1)}, w^{(2)}\right)\right) \Delta+L_{\epsilon} \Delta$ to the first and $\tilde{\Delta}$ to the second equation in (4.43) and spatial integration over $(0,1)$, we obtain for a.e. $t \in(0, \infty)$

$$
\begin{aligned}
\int_{0}^{1}\left(\left(\theta+\bar{\sigma}\left(w^{(1)}, w^{(2)}\right)\right) \Delta\right. & \left.+L_{\epsilon} \Delta\right) \Delta_{t}(x, t)+\tilde{\Delta}(x, t) \tilde{\Delta}_{t}(x, t)+\theta \Delta \tilde{\Delta}_{x}(x, t) d x= \\
& =\mu \int_{0}^{1} \tilde{\Delta}(x, t) \tilde{\Delta}_{x x}(x, t) d x
\end{aligned}
$$

In particular, due to (3.7),

$$
\begin{gathered}
\int_{0}^{1}\left(\theta+\bar{\sigma}\left(w^{(1)}, w^{(2)}\right)\right) \frac{d}{d t} \Delta^{2}+\frac{d}{d t} B_{\epsilon}(\Delta, \Delta)+\frac{d}{d t} \tilde{\Delta}^{2} d x \\
\leq \int_{0}^{1}-2 \mu \tilde{\Delta}_{x}^{2}+2 \theta|\Delta|\left|\tilde{\Delta}_{x}\right| d x .
\end{gathered}
$$

In the last relation, we have exploited the fact that, due to relation (2.6),

$$
\frac{d}{d t} B_{\epsilon}(\Delta, \Delta)=2 L_{\epsilon} \Delta \Delta_{t}
$$

Adding $2 \mu \tilde{\Delta}_{x}^{2}-2 \theta|\Delta|\left|\tilde{\Delta}_{x}\right|+\frac{\theta^{2}}{2 \mu} \Delta^{2} \geq 0$ to the right-hand side of the last relation gives for $K=\frac{\theta^{2}}{2 \mu}$

$$
\int_{0}^{1}\left(\theta+\bar{\sigma}\left(w^{(1)}, w^{(2)}\right)\right) \frac{d}{d t} \Delta^{2}+\frac{d}{d t} B_{\epsilon}(\Delta, \Delta)+\frac{d}{d t} \tilde{\Delta}^{2} d x \leq K \int_{0}^{1} \Delta^{2} d x .
$$

Due to Lemma 4.5 below, relation (4.44) implies for some $g \in L_{l o c}^{1}(0, \infty)$

$$
\frac{d}{d t}\left(\int_{0}^{1}\left(\theta+\bar{\sigma}\left(w^{(1)}, w^{(2)}\right)\right) \Delta^{2}+\tilde{\Delta}^{2} d x+B_{\epsilon}(\Delta, \Delta)\right) \leq g(t) \int_{0}^{1} \Delta^{2} d x .
$$

Note that, due to (4.31) and the boundedness of the bilinear form $B_{\epsilon}$ on $L^{2}(0,1) \times$ $L^{2}(0,1)$, we can choose $\theta>0$ such that

$$
\int_{0}^{1}\left(\theta+\bar{\sigma}\left(w^{(1)}, w^{(2)}\right)\right) \Delta^{2} d x+B_{\epsilon}(\Delta, \Delta) \geq \int_{0}^{1} \Delta^{2} d x
$$


Due to (4.45) and (4.46), we can apply Gronwall's lemma and obtain from (4.42) the desired uniqueness.

It remains to prove the following

Lemma 4.5. There exists a function $f \in L_{\text {loc }}^{1}(0, \infty)$ such that, for a.e. $x \in(0,1)$,

$$
\frac{d}{d t}\left(\bar{\sigma}\left(w^{(1)}(x, t), w^{(2)}(x, t)\right)\right) \leq f(t) .
$$

Proof: For $z \in \mathbb{R}$ define

$$
\mathfrak{S}(z)=\sigma(z)-\lambda z .
$$

Due to (4.48) and (3.2), the stress can be written in the following form:

$$
\sigma(w(x, t))+\left(L_{\epsilon} w\right)(x, t)=\mathfrak{S}(w(x, t))+\left(\hat{L}_{\epsilon} w\right)(x, t) .
$$

Recall the definition (4.31) of $\bar{\sigma}$ and assume that $\left(v^{(1)}, w^{(1)}\right)$ and $\left(v^{(2)}, w^{(2)}\right)$ are solutions of system (1.1).

Analogously as in (4.33) set

$$
q^{(i)}(x, y, t):=\mu\left(w^{(i)}(y, t)-w^{(i)}(x, t)\right)+\int_{y}^{x} v^{(i)}(z, t) d z .
$$

For $i=1,2$ we have a.e.

$$
\frac{d}{d t} q^{(i)}(x, y, t)=\sigma\left(w^{(i)}(x, t)\right)+\left(L_{\epsilon} w^{(i)}\right)(x, t)-\left(\sigma\left(w^{(i)}(y, t)\right)-\left(L_{\epsilon} w^{(i)}\right)(y, t)\right) .
$$

This relation follows analogously as the third identity in the last section for $\frac{d}{d t} q(x, y, t)$ on p.17.

For

$$
g^{(i)}(x, y, t)=\left(\hat{L}_{\epsilon} w^{(i)}\right)(x, t)-\left(\mathfrak{S}\left(w^{(i)}(y, t)\right)-\left(\hat{L}_{\epsilon} w^{(i)}\right)(y, t)\right)
$$

relation (4.51) can be equivalently written as

$$
\frac{d}{d t} q^{(i)}(x, y, t)=\sigma\left(w^{(i)}(x, t)\right)+g^{(i)}(x, y, t)
$$

Set

$$
b^{(i)}(x, y, t)=\frac{d}{d t}\left(\frac{1}{\mu} \int_{y}^{x} v^{(i)}(z, t) d z+w^{(i)}(y, t)+\frac{1}{\mu}\left(\hat{L}_{\epsilon} w^{(i)}(x, t)-\hat{L}_{\epsilon} w^{(i)}(y, t)\right)\right) .
$$


Then we obtain

$$
\begin{gathered}
\frac{d}{d t} \bar{\sigma}\left(w^{(1)}(x, t), w^{(2)}(x, t)\right)=\sum_{i=1}^{2} \bar{\sigma}_{z_{i}}\left(w^{(1)}(x, t), w^{(2)}(x, t)\right) w_{t}^{(i)}(x, t) \\
\stackrel{(4.34)}{=} \sum_{i=1}^{2} \bar{\sigma}_{z_{i}}\left(w^{(1)}(x, t), w^{(2)}(x, t)\right) \frac{d}{d t}\left(\frac{1}{\mu}\left[\int_{y}^{x} v^{(i)}(z, t) d z-q^{(i)}(x, y, t)\right]+w^{(i)}(y, t)\right) \\
\stackrel{(4.53)}{=} \sum_{i=1}^{2} \bar{\sigma}_{z_{i}}\left(w^{(1)}(x, t), w^{(2)}(x, t)\right)\left(b^{(i)}(x, y, t)+\frac{1}{\mu} g^{(i)}(x, y, t)-\frac{1}{\mu} \mathfrak{S}\left(w^{(i)}(x, t)\right)\right) .
\end{gathered}
$$

Due to $w^{(i)} \in C^{0}\left(0, \infty ; L^{2}(0,1)\right)$ and the Green's function estimates in the next section, there exists some $y \in[0,1]$ with the following property:

$$
b^{(i)}(x, y, \cdot), g^{(i)}(x, y, \cdot) \in L_{l o c}^{2}(0, \infty)
$$

Due to (4.32) and

$$
\mathfrak{S}(z)=z^{3}-(1+\lambda) z
$$

there exist finite real numbers $K_{i}=K_{i}\left(\left|c_{1}\right|,\left|c_{2}\right|,\left|c_{3}\right|,\left|c_{4}\right|\right.$ ) (being monotone increasing in each entry) with the following property:

$$
\sum_{i=1}^{2} \bar{\sigma}_{z_{i}}\left(z_{1}, z_{2}\right)\left(c_{1}+c_{2} \mathfrak{S}\left(c_{3}-c_{4} z_{i}\right)\right) \leq K_{1}+K_{2} c_{1}^{2} .
$$

From (4.55) and (4.56) the claim of Lemma 4.5 follows.

4.5. Green's function estimates. The aim of this section is to prove the following lemma:

Lemma 4.6. Assume that, for some $\mu>0, u=u(x, t)$ is the weak solution of

$$
u_{t}-\mu u_{x x}=f_{x} \text { in }(0,1) \times(0, \infty)
$$

to the following initial-boundary value problem:

$$
u(\cdot, 0)=u_{0} \in L^{2}(0,1), \quad u(0, t)=u(1, t)=0 \quad \forall t \in(0, \infty) .
$$

Furthermore, assume that, for some $q \in[1, \infty)$, the given right-hand side of equation (4.57) has the following properties:

$$
\begin{gathered}
f_{t} \in L^{2}((0,1) \times(0, \infty)), \\
f \in L^{\infty}\left(0, \infty ; L^{q}(0,1)\right)
\end{gathered}
$$


and, for any $\delta>0$,

$$
f \in L^{\infty}((0,1) \times(\delta, \infty)) .
$$

Then,

$$
u \in C^{0}\left([0, \infty) ; H^{1, q}(0,1)\right)
$$

and, for any $\delta>0$,

$$
u \in L^{\infty}\left(\delta, \infty ; H^{1, \infty}(0,1)\right) \quad \forall t \in(0, \infty) .
$$

Proof: For simplicity we assume that $\mu=1$. The case of general $\mu>0$ is analogous.

Fix any $t \in[0, \infty)$. Then, for any $\tau \in \mathbb{R}$ with $t+\tau \in[0, \infty), f(x, t+\tau)$ can be written in the following form:

$$
f(x, t+\tau)=f_{1}(t ; x)+f_{2}(t ; x, \tau)
$$

where

$$
f_{2}(t ; \cdot, 0) \equiv 0 .
$$

For notational convenience, we write $f_{1}(x)$ (resp. $f_{2}(x, \tau)$ ) instead of $f_{1}(t ; x)$ (resp. $\left.f_{2}(t ; x, \tau)\right)$.

From (4.59) we know that

$$
\left\|f_{2}(\cdot, \tau)\right\|_{L^{2}(0,1)} \leq K|t-\tau|^{\frac{1}{2}}
$$

where

$$
K=K\left(\left\|f_{t}\right\|_{L^{2}\left(0, \infty ; L^{2}(0,1)\right)}\right) .
$$

Assume we have shown that for the Green's function to (4.57) and (4.58) there exists a strictly positive real number $K$ with the following property:

$$
\left\|G_{x y}(x, y, t-\tau)\right\|_{L^{2}(0,1)} \leq K|t-\tau|^{-\frac{5}{4}} .
$$

Then, $u=u(x, t)$ can be written in the following form:

$$
u(x, t)=u_{1}(x, t)+u_{2}(x)-\int_{0}^{t} \int_{0}^{1} G_{y}(x, y, t-\tau) f_{2}(y, \tau) d y d \tau,
$$

where $u_{2}$ is the solution to the Poisson equation

$$
-\partial_{x x} u_{2}=\partial_{x} f_{1}
$$


with homogeneous Dirichlet boundary conditions

$$
u_{2}(0)=u_{2}(1)=0
$$

and $u_{1}$ is the solution of the following initial-boundary value problem:

$$
\partial_{t} u_{1}-\partial_{x x} u_{1}=0, \quad u_{1}(\cdot, 0)=u(\cdot, 0)-u_{2}, \quad u_{1}(0, t)=u_{1}(1, t)=0 .
$$

Derivation of (4.69) gives for $u_{x}(x, t)$ the following expression:

$$
u_{x}(x, t)=u_{1 x}(x, t)+u_{2 x}(x)-\int_{0}^{t} \int_{0}^{1} G_{y x}(x, y, t-\tau) f_{2}(y, \tau) d y d \tau .
$$

Due to (4.68), it follows

$$
\begin{gathered}
\left|\int_{0}^{t} \int_{0}^{1} G_{y x}(x, y, t-\tau) f_{2}(y, \tau) d y d \tau\right| \leq \int_{0}^{t} \int_{0}^{1}\left|G_{y x}(x, y, t-\tau) f_{2}(y, \tau)\right| d y d \tau \\
=\int_{0}^{t}\left\|G_{x y}(x, \cdot, t-\tau) f_{2}(\cdot, \tau)\right\|_{L^{1}(0,1)} d \tau \leq \int_{0}^{t}\left\|G_{x y}(x, \cdot, t-\tau)\right\|_{L^{2}(0,1)}\left\|f_{2}(\cdot, \tau)\right\|_{L^{2}(0,1)} d \tau \\
\leq \int_{0}^{t} K^{2}|t-\tau|^{-\frac{3}{4}} d \tau \leq \frac{1}{4} K^{2} t^{\frac{1}{4}} .
\end{gathered}
$$

From (4.70) it easily follows that

$$
\left\|u_{2 x}\right\|_{L^{q}(0,1)} \leq\left\|f_{1}\right\|_{L^{q}(0,1)},
$$

and, in case of $f_{1} \in L^{\infty}(0,1)$,

$$
\left\|u_{2 x}\right\|_{L^{\infty}(0,1)} \leq\left\|f_{1}\right\|_{L^{\infty}(0,1)} .
$$

Hence, the claim of Lemma 4.6 follows if we have shown relation (4.68).

In order to show relation (4.68) we recall that $G$ can be written as follows

$$
G(x, y, t)=\frac{1}{t^{\frac{5}{2}}}\left(G_{1}(x, y, t)+G_{2}(x, y, t)\right),
$$

where

$$
G_{1}(x, y, t)=\frac{t^{2}}{(4 \pi)^{1 / 2}} \sum_{n=-\infty}^{\infty} \exp \left(\frac{-(x-y+2 n)^{2}}{4 t}\right)
$$

and

$$
G_{2}(x, y, t)=-\frac{t^{2}}{(4 \pi)^{1 / 2}} \sum_{n=-\infty}^{\infty} \exp \left(\frac{-(x+y+2 n)^{2}}{4 t}\right) .
$$


As the series on the right-hand sides of (4.76) and (4.77) and their derivatives are uniformly convergent for any $t>0$, we may derive them term by term.

In particular,

$$
\partial_{x y} G_{1}(x, y, t)=\frac{1}{(4 \pi)^{1 / 2}} \sum_{n=-\infty}^{\infty}\left(\frac{t}{2}-\frac{(x-y+2 n)^{2}}{4}\right) \exp \left(\frac{-(x-y+2 n)^{2}}{4 t}\right)
$$

and

$$
\partial_{x y} G_{2}(x, y, t)=-\frac{1}{(4 \pi)^{1 / 2}} \sum_{n=-\infty}^{\infty}\left(\frac{t}{2}-\frac{(x+y+2 n)^{2}}{4}\right) \exp \left(\frac{-(x+y+2 n)^{2}}{4 t}\right)
$$

From (4.78) and (4.79) it is not hard to see that, for any

$$
(x, y, t) \in[0,1] \times[0,1] \times(0,1],
$$

$\left|G_{x y}(x, y, t)\right|$ can be majorized as follows:

$$
\left|G_{x y}(x, y, t)\right| \leq \frac{C}{t^{\frac{5}{2}}}\left(t+(x \pm y)^{2}\right) \sum_{i=1}^{4}\left|\mathcal{G}_{i}(x, y, t)\right|
$$

where

$$
\begin{aligned}
& \mathcal{G}_{1}(x, y, t)=\exp \left(\frac{-(x-y)^{2}}{4 t}\right), \\
& \mathcal{G}_{2}(x, y, t)=\exp \left(\frac{-(x+y)^{2}}{4 t}\right),
\end{aligned}
$$

$$
\mathcal{G}_{3}(x, y, t)=(x-y)^{2} \exp \left(\frac{-(x-y)^{2}}{4 t}\right)
$$

$$
\mathcal{G}_{4}(x, y, t)=(x+y)^{2} \exp \left(\frac{-(x+y)^{2}}{4 t}\right)
$$

and $C$ does not depend on $(x, y, t)$.

Due to (4.80), there exists a constant $\tilde{C}$ such that

$$
G_{x y}(x, y, t)^{2} \leq \frac{\tilde{C}}{t^{5}}\left(t^{2}+(x \pm y)^{4}\right) \sum_{i=1}^{4} \mathcal{G}_{i}(x, y, t)^{2} .
$$

From (4.85) we derive the estimate 


$$
\int_{0}^{1} \frac{1}{\sqrt{2} t^{1 / 2}} \exp \left(\frac{-(x \pm y)^{2}}{2 t}\right) d y \leq \int_{-\infty}^{\infty} e^{-y^{2}} d y<\infty
$$

and

$$
\int_{0}^{1} \frac{1}{\sqrt{2} t^{1 / 2}}\left(\frac{(x-y)^{4}}{4 t^{2}}\right) \exp \left(\frac{-(x \pm y)^{2}}{2 t}\right) d y \leq \int_{-\infty}^{\infty} y^{4} e^{-y^{2}} d y<\infty .
$$

Hence, $\int_{0}^{1} G_{x y}(x, y, t)^{2} d y=O\left(t^{-\frac{5}{2}}\right)$, or, in other words,

$$
\left\|G_{x y}(x, \cdot, t)\right\|_{L^{2}(0,1)}=O\left(t^{-\frac{5}{4}}\right)
$$

and relation (4.68) follows.

\section{Treatment of homogeneous Neumann boundary CONDition}

In order to consider the homogeneous Neumann boundary condition (1.4), let $v=$ $v(x, t)$ and $w=w(x, t)$ be weak solutions of system (1.1) with the following slight modifications on the domain of definition for $(x, t)$ and the operator $L_{\epsilon}$

$$
(x, t) \in[0,2] \times[0, \infty)
$$

(instead of $(x, t) \in[0,1] \times[0, \infty))$ and $L_{\epsilon} u=\hat{L}_{\epsilon} u-\lambda u$, where the convolution operator

$$
\hat{L}_{\epsilon} u(x)=\lambda \int_{0}^{1} \phi_{\epsilon}(x-y) u(y) d y, \quad \forall x \in[0,1]
$$

is extended by mirroring:

$$
\hat{L}_{\epsilon} u(1+x)=-\hat{L}_{\epsilon} u(1-x) \forall x \in(1,2] .
$$

We impose the Dirichlet boundary condition

$$
\int_{0}^{2} w(x, t) d x=\int_{0}^{2} w_{0}(x) d x
$$

where the initial values $\left(v_{0}, w_{0}\right)$ are assumed to have finite energy and to fulfill the following symmetry conditions:

$$
v_{0}(1+x)=v_{0}(1-x), \quad w_{0}(1+x)=-w_{0}(1-x) \text { for almost every } x \in(0,1)
$$

The proof of an analogous claim as in Theorem 3.1 for the modified operator $L_{\epsilon}$ goes along the lines of the proof of Theorem 3.1 and is left to the reader.

Furthermore, due to (5.89) and straighforward symmetry considerations, for the weak solution $(v, w)$ there holds for any $t \in(0, \infty)$ and almost every $x \in(0,1)$ :

$$
v(1+x, t)=v(1-x, t), w(1+x, t)=-w(1-x, t) .
$$


We will show the following lemma:

Lemma 5.1. If the initial values $\left(v_{0}, w_{0}\right)$ fulfill relation (5.89) and the initial strain $w_{0}$ is continuous at $x=1$ with

$$
\lim _{x \rightarrow 1} \sigma\left(w_{0}(x)\right)+\left(L_{\epsilon} w_{0}\right)(x)=0,
$$

then, for any $t \in[0, \infty), w(\cdot, t)$ is continuous at $x=1$.

Assume we have shown Lemma 5.1, then, due to (5.90), the homogeneous Neumann boundary condition is fulfilled:

$$
\lim _{x \rightarrow 1} \sigma(w(x, t))+\left(L_{\epsilon} w\right)(x, t)=0
$$

and we are ready with the proof of Theorem 3.1.

Proof of Lemma 5.1:

For convenience of the reader, we recall the definition

$$
q(x, y, t)=\mu(w(y, t)-w(x, t))+a(x, y, t),
$$

where

$$
a(x, y, t)=\int_{y}^{x} v(z, t) d z,
$$

and the identity following relation (4.36):

$$
\begin{aligned}
\frac{d}{d t} q(x, y, t)=\frac{\lambda}{\mu}(q(x, y, t) & -a(x, y, t))+\left(\hat{L}_{\epsilon} w\right)(x, t)-\left(\hat{L}_{\epsilon} w\right)(y, t) \\
+ & \sigma\left(\frac{1}{\mu}(a(x, y, t)-q(x, y, t))+w(y, t)\right)-\sigma(w(y, t)) .
\end{aligned}
$$

Due to the definition of $q$,

$$
w(x, t)=\frac{1}{\mu}[a(x, 1, t)-q(x, 1, t)]+w(1, t) .
$$

Due to (5.94) and $\lim _{x \rightarrow 1} a(x, 1, t)=0$, the continuity of $x \mapsto w(x, t)$ at $x=1$ is equivalent to the continuity of $x \mapsto q(x, 1, t)$ at $x=1$.

The continuity of $x \mapsto q(x, 1, t)$ at $x=1$ in turn follows from

$$
|q(x, 1, t)| \leq \int_{0}^{t}\left|g_{1}(x, s)\right||q(x, 1, s)|+\left|g_{2}(x, s)\right| d s \quad \forall t \in(0, \infty)
$$


and Gronwall's lemma, where, for any finite interval $[0, T]$, there exists some constant $C$ not depending on $x$,

$$
\left\|g_{1}(x, \cdot)\right\|_{L^{1}(0, T)}+\left\|g_{2}(x, \cdot)\right\|_{L^{1}(0, T)} \leq C
$$

and

$$
\lim _{x \rightarrow 1}\left\|g_{2}(x, \cdot)\right\|_{L^{1}(0, T)}=0 .
$$

In fact, relation (5.95) directly follows from (5.93): for

and

$$
g_{1}(x, t)=\frac{\lambda}{\mu}+\bar{\sigma}\left(\frac{1}{\mu}(a(x, 1, t)-q(x, 1, t))\right),
$$

$$
g_{2}(x, t)=\frac{1}{\mu} \bar{\sigma}\left(\frac{1}{\mu}(a(x, 1, t)-q(x, 1, t))\right) a(x, 1, t)-\frac{\lambda}{\mu} a(x, 1, t)+\left(\hat{L}_{\epsilon} w\right)(x, t)
$$

(where $\bar{\sigma}$ is given by (4.31) and $\hat{L}_{\epsilon}$ is the convolutional part of $L_{\epsilon}$ ) we have

$$
\frac{d}{d t} q(x, 1, t)=g_{1}(x, t) q(x, 1, t)+g_{2}(x, t) .
$$

Then, relation (5.96) is straightforward.

As $t \mapsto v(\cdot, t)$ is uniformly bounded in $L^{2}(0,1)$, we have

$$
\lim _{x \rightarrow 1} a(x, 1, t)=\lim _{x \rightarrow 1} \int_{x}^{1} v(z, t) d z=0 \text { uniformly in } t \text { for } t \in[0, T] .
$$

The last relation and the assumptions on the convolution kernel in $\hat{L}_{\epsilon}$ imply relation (5.97).

\section{REFERENCES}

[1] G. Alberti \& G. Bellettini, A non-local anisotropic model for phase transitions: Asymptotic behaviour of rescaled energies, European J. Appl. Math. 9, No. 3, 261-284 (1998)

[2] G. Andrews, On the existence of solutions to the equation $u_{t t}=u_{x x t}+\sigma\left(u_{x}\right)_{x}, J$. Differential Equations 35, 200-231 (1980)

[3] G. Andrews \& J.M. BALL, Asymptotic behaviour and changes of phase in one-dimensional nonlinear viscoelasticity, J. Differential Equations 44, 316-341 (1982)

[4] S. Demoulini, Weak solutions for a class of nonlinear systems of viscoelasticity, Arch. Rat. Mech. Anal. 155, 299-334 (2000)

[5] A. Dressel \& C. Rohde, Time-asymptotic behaviour of solutions for a viscoelastic two-phase model with nonlocal capillarity, in preparation.

[6] A.C.Eringen, Nonlocal continuum field theories, Springer, 2002

[7] G. Friesecke, G. Dolzmann, Implicit time discretization and global existence for a quasi-linear evolution equation with nonconvex energy, SIAM J. Math. Anal. 28, No. 2, 363-380 (1997)

[8] K.-H. Hoffmann \& P. RYBKa, On convergence of solutions to the equation of viscoelasticity with capillarity, Commun. in Partial Differential Equations 25, 1845-1890 (2000) 
[9] L. ModicA, The gradient theory of phase transitions and the minimal interface criterion, Arch. Rat. Mech. Anal. 98, 123-142 (1987)

[10] R. Rogers \& L. Truskinovsky, Discretization and hysteresis, Phys. B 233, 370-375 (1997)

[11] X. Ren \& L. Truskinovsky, Finite scale microstructures in nonlocal elasticity, Journal of Elasticity 59, 319-355 (2000)

[12] C. RohDE, Phase transitions and sharp-interphase limits for the 1d-elasticity system with nonlocal energy, Interfaces and Free Boundaries 7, 107-129 (2005)

[13] C. Rohde \& M.D. Thanh, Global Existence for Phase Transition Problems via a Variational Scheme, J. Hyp. Differential Equations 1, No. 4, 747-768 (2004)

Institut für Angewandte Analysis und Numerische Simulation, Universität Stuttgart, Pfaffenwaldring 57, 70569 Stuttgart, Germany

EMAIL:ALEXANDER.DRESSEL|CHRISTIAN.ROHDE@MATHEMATIK.UNI-STUTTGART.DE 